\title{
Evolutionary dynamics in the fungal polarization network, a mechanistic perspective
}

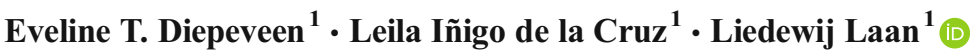

Received: 3 May 2017 / Accepted: 24 July 2017 / Published online: 15 August 2017

(C) The Author(s) 2017. This article is an open access publication

\begin{abstract}
Polarity establishment underlies proper cell cycle completion across virtually all organisms. Much progress has been made in generating an understanding of the structural and functional components of this process, especially in model species. Here we focus on the evolutionary dynamics of the fungal polarization protein network in order to determine general components and mechanistic principles, species- or lineage-specific adaptations and the evolvability of the network. The currently available genomic and proteomic screens in a variety of fungal species have shown three main characteristics: (1) certain proteins, processes and functions are conserved throughout the fungal clade; (2) orthologous functions can never be assumed, as various cases have been observed of homologous loci with dissimilar functions; (3) species have, typically, various species- or lineage-specific proteins incorporated in their polarization network. Further large-scale comparative and experimental studies, including those on nonmodel species representing the great fungal diversity, are needed to gain a better understanding of the evolutionary dynamics and generalities of the polarization network in fungi.
\end{abstract}

Keywords Cell polarity · Protein network - Evolution · Fungi $\cdot$ Evolutionary conservation $\cdot$ Adaptation

This article is part of a Special Issue on 'IUPAB Edinburgh Congress' edited by Damien Hall.

Liedewij Laan

1.laan@tudelft.nl

1 Department of Bionanoscience, Kavli Institute of NanoScience, Faculty of Applied Sciences, Delft University of Technology, P.O. Box 5046, 2600 GA Delft, the Netherlands

\section{Introduction}

The asymmetrical accumulation of cellular components, such as organelles, proteins and cell-wall components, is fundamental for a proper completion of the cell cycle in both unicellular and multicellular organisms (Drubin 2000). The crucial step of symmetry breaking is observed during various cellular processes, such as cell division, cell motility, cell differentiation, cell-cell signaling and cell fusion (see, for example, Li and Bowerman 2010). The process of how cells break symmetry and polarize has been the focus of much attention in the literature and has been studied for over five decades. Polarity establishment has been traditionally studied in the budding yeast Saccharomyces cerevisiae. Budding yeast cells need to break symmetry, such as during each cell cycle in order to form a site of polarized cell growth and establish a bud that develops into a daughter cell. Much progress has been made in disentangling the structural and functional aspects of the components of this fundamental protein network. This wealth of information provides a valuable backbone for testing hypotheses on homology and for examining levels of conservation and divergence of the protein network members across different species. The remarkably diverse kingdom of fungi provides great opportunities to perform such comparisons, as it comprises an astonishing number of relative closely related species with a wide variety of cellular morphologies. The availability of various model organisms, such as the fission yeast Schizosaccharomyces pombe, the filamentous ascomycete Ashbya gossypii and the pathogenic basidiomycete Ustilago maydis or corn smut, facilitates direct comparisons of the functional and structural components of the machinery for cell polarity.

In this review we present: (1) an overview of the polarization machinery in the main fungal model species, (2) a discussion on the evolutionary dynamics of the network in fungi and 
(3) an overview of the current mechanistic understanding of the polarization machinery. Finally, we aim to link the discussed patterns of diversity, conservation and function to the great organismal diversity that we observe all around us, and recommend future directions of study.

\section{The polarization machinery of fungal model systems}

The establishment of cell polarity is initiated by intrinsic or extrinsic cues, such as the marked site of previous cell division or mating pheromones in yeast, and results in the asymmetrical distribution of cell content. Polarity is essential to numerous processes during embryonic development, neural development and immunity throughout the Eukaryota (Chant 1999). In yeast this subcellular asymmetry is essential for proper cell division (e.g. budding) and mating (Chant 1999). Cellular content needs to be rearranged and allocated to the site of polarization during these processes. Actin patches and cables combined with myosin proteins facilitate this allocation and subsequent regional expansion. Proteins, vesicles, organelles and the cytoskeleton all relocate to the site of cytokinesis or mating. The nucleus (or nuclei, in the case of filamentous fungi) is oriented towards that site by microtubules. Two important steps are distinguished: (1) determining the direction of polarization, based on, for example, landmark proteins, pheromones, uneven distribution of mRNA; (2) the further establishment of the axis of polarization through activation of protein networks regulated by GTPases (Fig. 1). After these steps cell growth and subsequent cell division or mating take place. The signaling pathways involved in regulating these processes are hypothesized to be ancient and conserved, as they are found in all eukaryotes (Pruyne and Bretscher 2000). Molecular key players in these protein networks have been identified and characterized in detail in the budding yeast Saccharomyces cerevisiae (Table 1). Various excellent reviews, commentaries and seminal works are available on the details of budding yeast's polarization protein networks (Pringle et al. 1995; Chant 1999; Pruyne and Bretscher 2000; Drees et al. 2001; Chang and Peter 2003; Pruyne et al. 2004; Park and Bi 2007; Bi and Park 2012; Martin and Arkowitz 2014). Here we give a brief overview of the main proteins, their functions and the main processes involved (Table 1).

Landmark formation is a crucial step and depends on the state of the cell; haploid cells make use of the axial patterning proteins (i.e. Axl1, Axl2, Bud3, Bud4; Chant and Herskowitz 1991; Fujita et al. 1994; Halme et al. 1996; Roemer et al. 1996), while diploid cells make use of the bipolar patterning proteins (i.e. Bud8, Bud9, Rax1, Rax2; Zahner et al. 1996; Chen et al. 2000; Fujita et al. 2004). Both types of cells require a shared group of proteins consisting of Rsr1, Bud2, Bud5 (Bender and Pringle 1989; Chant et al. 1991; Chant and

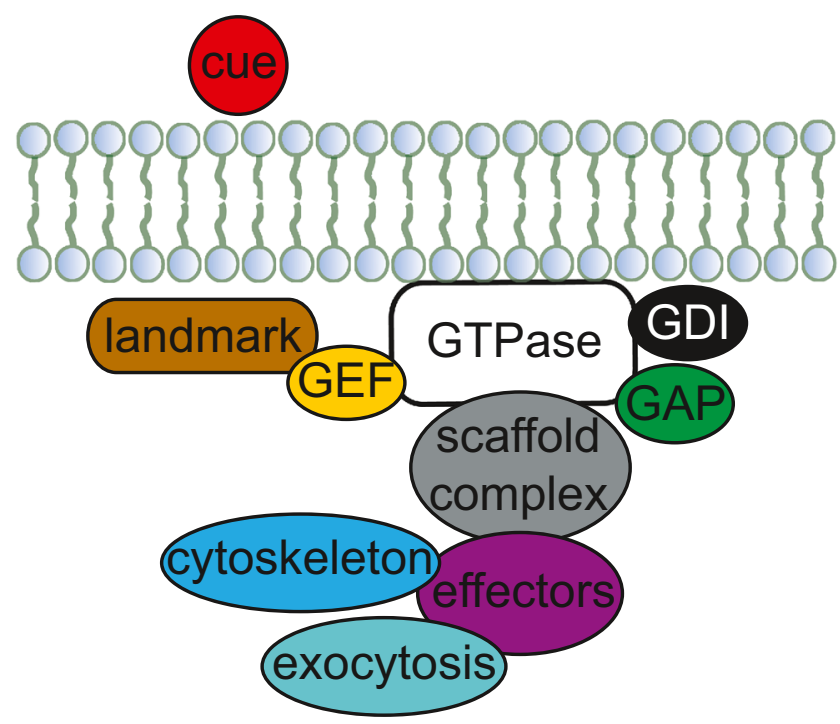

Fig. 1 The key network of polarization. This cartoon gives an overview of the functional steps of cellular polarization. Briefly, polarity establishment is initiated by external or internal cues (shown here as an extrinsic cue; in red) resulting in landmark formation (landmark, in brown). The key regulator of polarity establishment and maintenance is a GTPase, which is regulated by its guanine nucleotide exchange factors $(G E F$, in yellow), GTPase-activating proteins (GAP, in green) and guanosine nucleotide dissociation inhibitors (GDI, in black). GTPase effectors (effectors, in purple) form a link between the scaffold protein complex (scaffold complex, in gray) and downstream processes, such as cytoskeleton organization (cytoskeleton, in turquoise) and exocytosis (in blue). For more information about the proteins involved in each step see Table 1. Note that the size of the circles do not represent protein concentrations in the cell or absolute protein (complex) sizes

Herskowitz 1991; Park et al. 1993). This landmark establishment acts as a spatial memory of the precise location for the next cell cycle. Subsequently, there is a direct link between the landmark system and key regulators of polarization: the GTPase Cdc42 and its activator the guanine nucleotide exchange factor (GEF) Cdc24 (see Chant 1999 for hypotheses on proposed interactions). The signal transduction system for determining the polarization axis during mating is initiated by mating pheromones and consists of various proteins, such as Ste20, Ste5, Ste11, Ste7, Fus3, Ste12 and Far1 (Peter and Herskowitz 1994; Herskowitz 1995; Whiteway et al. 1995; Inouye et al. 1997; Leeuw et al. 1998; Pryciak and Huntress 1998). Observations of the authors of these studies reveal that different cues for different cell types exist and that these initiate different protein signal transduction pathways.

The polarization axis is still determined with equal efficiency even when mating or landmark cues are unavailable (e.g. Drubin 2000; Smith et al. 2013). Symmetry breaking can be kick-started by small fluctuations in concentrations away from homogeneity, which would otherwise be overruled by the aforementioned spatial cues. A switch-like mechanism controlled by the cyclin-dependent kinase $\mathrm{Cdc} 28$ then prevents untimely amplification of these ever-present fluctuations (Gulli et al. 2000; Li and Bowerman 2010; Klünder et al. 
Table 1 Overview of polarization proteins across fungal model systems

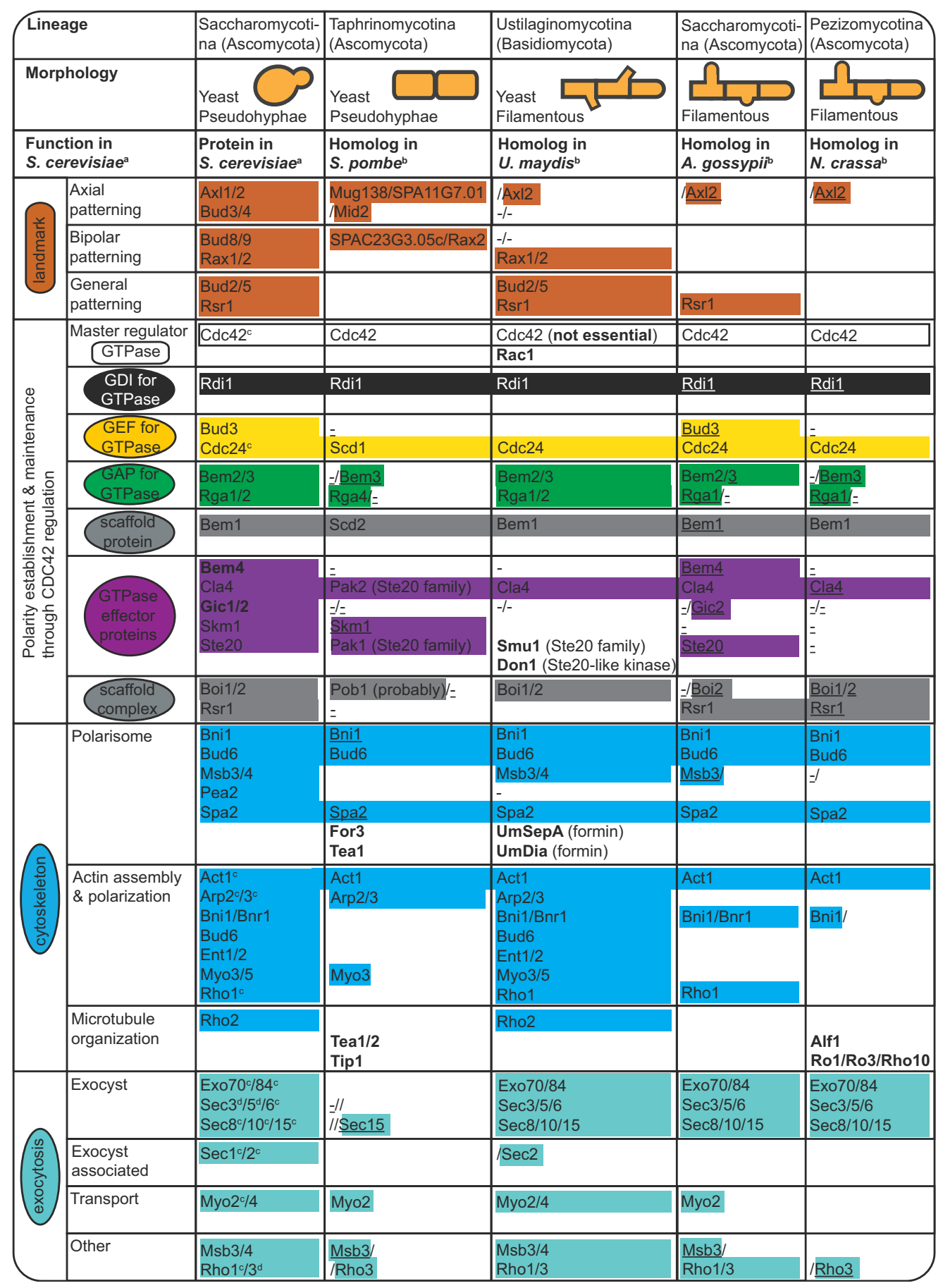

Proteins in bold represent species/lineage-specific polarization proteins and/or polarization proteins not found in S. cerevisiae. The minus symbol (-) represents proteins' absence in examined studies. Empty lines represent cases for which no information was found in the examined literature. This synopsis is not exhaustive

${ }^{a}$ Following SGD; see main text for further references. Overall functional groupings (1st \& 2nd column) as in Fig. 1. S. cerevisiae essential polarization proteins, Cdc28, Iqg1, Sec4 are non-evolvable (Liu et al. 2015)

${ }^{\mathrm{b}}$ Following (Feierbach and Chang 2001; Pelham and Chang 2002; Chang and Peter 2003; Harris 2006; Banuett et al. 2008; Kaufmann 2008; Köhli et al. 2008; Bi and Park 2012; Martin and Arkowitz 2014); see main text for further references. Underlined (absence of) proteins represent findings of our recent survey (Diepeveen et al. 2017)

${ }^{\mathrm{c}}$ Non-evolvable essential locus (Liu et al. 2015)

${ }^{\mathrm{d}}$ Evolvable essential locus (Liu et al. 2015) 
2013). For an overview of biophysical details see Box 1 in the Appendix.

To allocate cellular components to the site of budding or mating, the main axis for polarization then needs to be established and maintained, followed by polarization of the cytoskeleton. One of the most important steps is the localization and polarization of the master regulator of polarization, the GTPase Cdc42. Cdc42 interacts with many different proteins, including its own regulators (see, for example, Chant 1999; Martin 2015 for descriptions of the complex interconnectivity of Cdc42; Fig. 1; Table 1). There are three different groups of $\mathrm{Cdc} 42$ regulators: the GEFs (e.g. Cdc24 and the recently discovered Bud3) (Chant 1999; Pruyne and Bretscher 2000; Kang et al. 2014); the guanosine nucleotide dissociation inhibitors (GDI; e.g. Rdi1) (see Pruyne and Bretscher 2000; Martin 2015); GTPase-activating proteins (GAPs; e.g. Bem2, Bem3, Rga1, Rga2) (Kim et al. 1994; Zheng et al. 1994; Pringle et al. 1995; Stevenson et al. 1995; Chen et al. 1996; Chant 1999; Pruyne and Bretscher 2000; Drees et al. 2001; Smith et al. 2002; Chang and Peter 2003; Pruyne et al. 2004; Park and Bi 2007; Bi and Park 2012; Martin and Arkowitz 2014). These proteins control the (in)activation of active $\mathrm{Cdc} 42$ and dissociation from the cell membrane. Together with the important scaffolding protein Bem1, which mediates complex formation with Cdc42 and its GEF Cdc24, and, for example, Rsr1, these regulators help to spatially restrict the accumulation of active Cdc42 (Chant and Herskowitz 1991; Chenevert et al. 1992; Fujita et al. 1994; Zheng et al. 1995; Halme et al. 1996; Roemer et al. 1996; Park et al. 1997; Klünder et al. 2013). Cdc42 also interacts directly with a group of so-called effector proteins (Table 1). This group consists of Cla4, Ste20, Gic1, Gic2, Skm1 and Bem4. Bem4 interacts with various proteins, including Gic1, possibly localizing or regulating GTPases to the bud neck, and the major regulator of cell polarity Rhol (see Zahner et al. 1996; Chen et al. 2000; Drees et al. 2001; Fujita et al. 2004). The polarization pathway has to stimulate the cytoskeleton and secretion systems to complete polarized cell growth. Boil/2 are ligands of Bem1 and interact with various other proteins, such as Msb1 and Rho3, potentially forming a link between $\mathrm{Cdc} 42$ and proteins involved in, for example, exocytosis (Bender and Pringle 1989; Chant et al. 1991; Chant and Herskowitz 1991; Park et al. 1993; Matsui et al. 1996; Bender et al. 1996; Liao et al. 2013). The effector proteins Gic1 and Gic2 contribute to the polarization of the cytoskeleton (Chen et al. 1997; Brown et al. 1997; Chant 1999). Additional proteins connecting the polarization pathway to the cytoskeleton are, for example, Rho3, which directly targets Myo2 (Peter and Herskowitz 1994; Herskowitz 1995; Whiteway et al. 1995; Inouye et al. 1997; Leeuw et al. 1998; Pryciak and Huntress 1998; Robinson et al. 1999), and the Arp2/3 complex, which is involved in actin filament assembly (Machesky et al. 1994). The transportation of secretory vesicles to the site of polarization is controlled by a protein complex called the exocyst, consisting of, for example, Sec3 for membrane docking of vesicles and interaction with Rho1 (see, for example, Chant 1999). Interactions have been found for Rsr1, Bem1, Exo84 and Cde24 with the exocyst subunit Sec15 (Drees et al. 2001). These complex protein-protein interactions ensure a proper establishment and maintenance of cell polarity.

Interestingly, the fundamental significance of budding yeast's polarization machinery is supported by the identification of homologous proteins in non-fungal eukaryotes. The best example of homology, high-sequence similarity and functional conservation between distantly related species, is observed for $\mathrm{Cdc} 42$. This key regulator of polarization has been identified in hominids, rodents, teleost fishes and invertebrates, such as nematodes and fruit flies, among others (Johnson 1999; Cotteret et al. 2002). The signaling pathways underlying polarization are hypothesized to be common to all Metazoa. The PAR proteins, discovered in the nematode Caenorhabditis elegans (Kemphues et al. 1988), are conserved throughout the Metazoa (Goldstein and Macara 2007). The six C. elegans PAR proteins together with PKC3 and $\mathrm{Cdc} 42$ form a signaling network that is responsible for the asymmetric cell division observed in C. elegans zygotes (Goldstein and Macara 2007). Orthologous proteins for this network (with the exception of PAR2) have been identified in Drosophila melanogaster. A distant resemblance was only found for two PAR proteins in budding yeast (Goldstein and Macara 2007). A vertebrate GEF for Cdc42 has also been identified (Nishimura et al. 2006). These studies show that Cdc42 plays a major role in the establishment of polarization across a wide variety of organisms and therefore has an ancient origin.

The polarization networks of various species of fungi have been examined (Table 1). Homologous proteins can be detected based on sequence similarity in respect to, for example, budding yeast proteins by means of genomic and/or proteomic screens, but orthologous functions of such proteins cannot be ensured without functional characterization. Various phenotypical distinctions, which potentially affect the polarization network and protein repertoire, can be made between species (e.g. unicellular vs. multicellular organism, symmetric growth, isotropic growth or asymmetric growth with a budding site; Fig. 2). The unicellular fission yeast Schizosaccharomyces pombe provides an excellent starting organism for comparison to determine the resemblances in cell polarity establishment between unicellular species. In both $S$. pombe and $S$. cerevisiae a specific site on the cell membrane is marked by landmark proteins in response to a cue, i.e. small GTPase proteins which are translocated to this spot and regulate actin cables and microtubules polarization towards the site of future growth. The new site of polarization is initiated based on the previous site of cell division in both 
S. cerevisiae

Asymmetric growth, budding

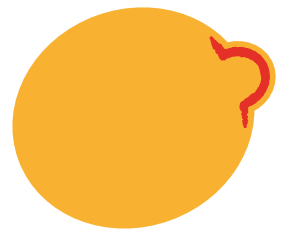

S. pombe

Symmetric growth, fission

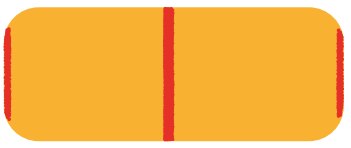

N. crassa - U. maydis

A. gossypii

Apical growth, division
Fig. 2 Overview of the different modes of polarization in different fungal cellular morphologies. Schematic representations of a budding yeast (left), fission yeast (center) and filamentous fungal (right) cell (or part thereof) are shown. The regions where polarization proteins accumulate during polarity establishment and cell division are highlighted in red. Saccharomyces cerevisiae forms a protrusion, referred to as a bud, at one side of the cell, which develops into a daughter cell. Schizosaccharomyces pombe grows at cell tips. Cells divide by medial

species. Homologs of budding yeast's landmark proteins Rax 1/2 (SPAC23G3.05c/Rax2) have been identified in fission yeast, as have homologs for the axial budding proteins Bud4 (Mid2), Axl1 (Mug138) and Axl2 (SPA11G7.01) (see Martin and Arkowitz 2014; Table 1). Cdc42 is required for the establishment of cell polarization, and the Cdc24 ortholog Scd1 is the fission yeast's GEF, with Gef1 as the second GEF (Coll et al. 2003). The Bem1 ortholog Scd2 might act as a scaffolding protein, although its exact role is not entirely clear (Chang et al. 1994). The Pob1 scaffolding protein has similarities to Boi1/2 and localizes For3 and the exocyst (Rincon et al. 2009; Nakano et al. 2011). Rdil is also present in fission yeast (see Martin and Arkowitz 2014). Although four different GAPs have been identified in budding yeast, only one GAP, Rga4, has previously been found in fission yeast, but in a recent survey we also identified a protein with homology to Bem3 (Diepeveen et al. 2017). Cdc42 effectors are the Ste20 family proteins Pak1/2 (Martin and Arkowitz 2014).

The fission yeast's proteins Tea1, Tea2 and Tip1 have not been found in Saccharomyces cerevisiae's symmetry breaking, but they do play critical roles in the spatial microtubule organization that marks the cell poles in Schizosaccharomyces pombe (Browning et al. 2000; Brunner and Nurse 2000). The Ras1 pathway plays an important role in regulating the polarization pathway. Ras 1 activates Cdc42 (Martin and Arkowitz 2014). The fission yeast's protein complex polarisome consists of Tea1, formin For3 and Bud6, with possible connections between For 3 and $\mathrm{Cdc} 42$ and Rho3 (Chang and Peter 2003). Putative effectors of Tea 1 are Pom 1 and Tea 3 (Nakano et al. 2002). Budding yeast has two formins, Bnil and Bnrl, involved in the assembly of actin cables and rings (Evangelista et al. 1997). One specific aspect of cytokinesis that is substantially different between budding and fission yeasts is that in fission yeast the site of cell division is in the center of the cell and not at the periphery of the cell. Mid1 is a candidate as landmark protein for the positioning of the nucleus and contractile ring assembly (Chang and Peter 2003). These studies show that there are some striking similarities in fission to produce two daughter cells. The filamentous species grow at their tips, and cells are formed by septum formation (not shown). Note that $S$. cerevisiae and $S$. pombe are dimorphic and are able to form pseudohyphae in addition to the unicellular yeast state depicted here. For the dimorphic Ustilago maydis we only depict the filamentous state and ignore the budding yeast-like state this species has during the haploid phase

key components and the core mechanism of the polarization network between the two yeast models, but at the same time the species are characterized by different modes of growth and cell division, which affects polarity establishment.

Non-yeast fungal species, such as Ustilago maydis, Ashbya gossypii and Neurospora crassa, have also been examined as key players and mechanisms of cell polarization (see Table 1). A remarkable difference between unicellular yeast (-like) species and filamentous fungi is that the latter are characterized by continuous polarized growth at the tips of their hyphae, thereby displaying specialized physiological aspects, such as tip-high calcium gradients (see, for example, Jackson and Heath 1993). The corn smut $U$. maydis is characterized by four possible states of polarized growth: yeast-like budding, filamentous structures in response to pheromones, tip-growth of newly formed dikaryotic hyphae outside the plant host and true filamentous structures inside the plant (see Banuett et al. 2008 and references therein). This fungus contains parts of the protein networks for, as an example, the recognition and interpretation of the landmark system (e.g. Axl2, Rax1/2, Rsr1, Bud2/5), polarity establishment (homologs of, for example, Cdc42, Cdc24, Bem3, Rga1/2, Cla4), exocytosis (e.g. Sec3/5/6/8/10/15) and actin (e.g. Arp2/3) and microtubule organization (e.g. Rho2) (Banuett et al. 2008). Interestingly budding yeast proteins such as Bud3/4/8/9 and Gic1/2 are missing, supporting earlier findings that these proteins are lineagespecific and restricted either to the Ascomycota or to the Saccharomycetaceae family (Diepeveen et al. 2017). Banuett also observed several polarization proteins not present in Saccharomyces cerevisiae; namely, the Rho GTPase Rac1, homolog of the mammalian Rac, has partially overlapping functions with $\mathrm{Cdc} 42$ in other filamentous fungi (see Banuett et al. 2008 for references) and is required for polarized growth. Even though homologs of proteins with functions in cell polarity in budding yeast are observed in U. maydis, they are not always characterized by orthologous functions. Most importantly, Cdc42 is not required for polarized growth in U. maydis (Mahlert et al. 2005). 
The filamentous plant pathogen Ashbya gossypii also shows continuous growth at the tips of the filaments. New sites of polarization are initiated during hyphal growth, resulting in multiple polarity axes simultaneously (see Philippsen et al. 2005). Its genome has been screened for Saccharomyces cerevisiae homologs. One particular protein important for maintenance of hyphal growth, albeit not essential in A. gossypii, is Rho3 (Wendland and Philippsen 2001). The same authors also identified the presence of $\mathrm{Cdc} 42$, Cdc24, Rho1 (Wendland and Philippsen 2001), Bem2 (Wendland and Philippsen 2000) and Rsr1 (Bauer et al. 2004) in A. gossypii. Mutants for these loci show altered patterns or loss of polarity. Mutants of the polarisome proteins Bni1 and Spa2 also result in unusual patterns of growth and hyphal development, as discussed in Philippsen et al. (2005). Again, even though a group of $S$. cerevisiae proteins is found in A. gossypii, clear differences have been observed in how cells make use of them during symmetry breaking (see Philippsen et al. 2005). A recent study has found that the polyQ-containing protein Whi3 is an important regulator of symmetry breaking in A. gossypii (Lee et al. 2015). Whi3 aggregates and forms RNA-protein assemblies with Bni1 and Spa2 RNA molecules. This results in the asymmetrical synthesis of these important polarization proteins, thereby promoting symmetry breaking events.

Mutant screens in the red bread mold Neurospora crassa have lead to the identification of important elements that contribute to polarized growth in this ascomycetous fungus. These proteins are involved in, for example, actin (e.g., Act1) or microtubule cytoskeleton (i.e. Alf1, Ro1, Ro3, Ro10), components of signaling pathways (e.g. Cdc42, Cdc24, Bem1, Lrg1) and the secretory pathway (e.g. Sar1, Sec53) (Seiler and Plamann 2003). Interestingly, mutants show distinct differences in phenotype (e.g. overall or local effects) which have not been detected in unicellular yeast. Seiler and Plamann (2003 hypothesized that filamentous growth is more complex and has a more dynamic regulatory regime. These same authors also postulated that in light of $N$. crassa's fast and constant hyphal growth at the tip, the secretory system is especially important in this species and the microtubular network is more complex. Homologs of budding yeast's polarisome have been found, namely, Spa2, Bni1, Bud6 and also the exocyst components Sec3, 5, 6, 8, 10, 15 and Exo70,84 (see Riquelme et al. 2011). Various studies, especially those in $N$. crassa, stress the complex differences between model organisms with different phenotypes.

It has often been hypothesized that at the very least the overall mechanistic principles and cellular processes observed in Saccharomyces cerevisiae are conserved throughout the fungal clade and across the Eukaryota (Pruyne and Bretscher 2000; Wendland 2001; Nelson 2003). The overview in Table 1 illustrates this by means of various proteins present in all of the examined fungal model organisms. Work covering a broad spectrum of fungal species has focused on detecting orthologous of $S$. cerevisiae polarization proteins. Nevertheless, our brief overview of the mechanisms in various model species indicates that there are various vast differences in how organisms make use of the S. cerevisiae orthologs. All model species also have lineage- or species specific proteins that are not found in the other species (Table 1; Diepeveen et al. 2017), indicating a level of unique specialization. Recent progress in, for example, integrating molecular data into theoretical modeling (Box 1 in the Appendix) suggest a possibility of multiple polarization networks, which are hypothesized to be implemented differently in different species (Goryachev and Leda 2017). Although detailed and large-scale genetic and mutant screens are available in few fungal model species (Neurospora crassa: Seiler and Plamann 2003; Aspergillus nidulans: Li et al. 2006), further examination across nonmodel fungal species is needed to determine a more precise degree of homology. Other approaches that ultimately provide valuable insights are currently being developed, such as largescale orthology prediction tools (T. Gehrmann, personal communication), the creation of genome-wide knock-out libraries and experimental studies, including experimental evolution and synthetic biology studies (minimal system, cell-rewiring to discover alternate or "bypass" mechanism). We currently have many individual and/or small-scale comparative findings, but an overall and detailed digest on the functionality, variability and conservation of the symmetry breaking mechanism across many species is still lacking.

\section{Evolutionary dynamics of the polarization protein network}

How complex patterns are formed and how individuals adapt to their environments are key questions in cell and evolutionary biology. It has recently become possible to combine these questions and ask if, and if so, how the biomolecular networks that form these complex patterns affect the adaptation of an organism to its environment. With the availability of a variety of resources, such as genome databases, strain collections and gene-deletion databases, the budding yeast Saccharomyces cerevisiae and its close relatives are great model systems to study this topic. Questions related to how strains or species adapt to specific environments, especially members of the Saccharomycetaceae family, range from experimental evolution studies on laboratory strains (Segrè et al. 2006; Leu and Murray 2006; Gresham et al. 2008) to specifying characteristics, such as performance of wild species in fermentation (Boynton and Greig 2016). In relation to the fungal polarization network, our previous work focused on the evolutionary dynamics of the polarization network both within budding yeast and across the fungal phylogeny (Laan et al. 2015; Diepeveen et al. 2017; discussed in more detail in the 
following text). By combining the findings of the different approaches our aim here is to reveal patterns of evolutionary dynamics in this biomolecular network.

Various factors and processes that influence protein (network) evolution have been described (see, for example, Pál et al. 2006; Evlampiev and Isambert 2008; Zhang and Yang 2015 for a discussion of these factors). One group of causal factors is generated by the functional importance of proteins. Interestingly, the full Saccharomyces cerevisiae genome has been examined and tested for essential genes (Liu et al. 2015), with the results providing insights into whether a cell is viable or not without the locus under study. Essentiality is, however, strongly dependent on the genetic background and can be different not only in different strains or species, but also in different environmental conditions, such as growth medium. Liu et al. screened essential proteins for cellular evolvability (Liu et al. 2015). They found that approximately $9 \%$ of the approximately 1000 proteins identified as essential could in fact overcome the initial loss by adaptive evolution within 10 days. Various proteins connected to the polarization network are found to be essential in S. cerevisiae (Table 1) and are involved in different steps during symmetry breaking (see Fig. 1), such as timing (Cdc28), the key-regulator of polarization $\mathrm{Cdc} 42$, and its GEF Cdc24, actin assembly (Act1, Arp2/3) and exocytosis (Sec3/5/6/8/10/15, Exo70/84, Rho1/3). Most of these essential proteins are non-evolvable, and only Rho 3 and Sec $3 / 5$ mutants can recover under standard conditions. These results indicate that evolvability is very different between members of the same cellular protein network.

In previous work we investigated the evolvability of the polarization network by examining how the polarization protein adapted to a strong genetic perturbation - in this case by means of inactivation of the nearly essential scaffolding protein Bem1. By deleting Bem1 we focused on adaptation to this specific cellular module and created a model system to obtain a mechanistic understanding of adaptation. First, we found that polarization is heavily disturbed in this model, with, as a result, decreases in growth rates by roughly eightfold. Bem1 binds directly to $\mathrm{Cdc} 42$ and forms the Bem1/Cdc42 complex by recruiting further proteins (see section The polarization machinery of fungal model systems; Lin et al. 2009). With Bem1 inactivated, the activation of $\mathrm{Cdc} 42$ by the GEF Cdc24 is hypothesized to be reduced. Interestingly, we found that yeast cells overcame this detrimental effect by inactivation of the GAPs Bem3 and Bem2, thereby reducing the inactivation of the available $\mathrm{Cdc} 42$. Another mechanism hypothesized to act at the same time is the inactivation of Nrp1, which was also observed. Nrp1 has a presumed function in the timing and initiation of polarization (Laan et al. 2015), which is brought forward by the actions of $\operatorname{Cdc} 28$ (Lew and Reed 1993). This protein stimulates the release of $\mathrm{Cdc} 24$ from the nucleus, which in turn activates $\mathrm{Cdc} 42$ (Shimada et al. 2000). These results indicate that the polarization protein network can adapt to perturbations within a few hundred generations and that it adapts by altering the regulation of the key regulator of polarization- $\mathrm{Cdc} 42$. As such, it would appear that the polarization network of Saccharomyces cerevisiae can overcome perturbations and relatively rapidly adapt to changes to key components by regulatory shifts.

We found that the polarization network is able to adapt under laboratory conditions. To study the relevance for evolution in the wild we asked how conserved the budding yeast's polarization repertoire is across the fungal tree of life. In an attempt to answer this question we screened 200 fungal strains and species from four different phyla for 34 selected polarization proteins. We observed dynamic patterns in the sheer number of proteins per strain/species, in the sequence similarity of the proteins and in the overall prevalence of proteins (Diepeveen et al. 2017). The examined strains and species grouped into three main clusters (i.e. filamentous fungi, basal unicellular fungi and yeast-like fungi) based on protein repertoire size, lineage, lifestyle and genetic distance. There was a reduction in repertoire size in the filamentous basidiomycetes and a further reduction in the basal and unicellular lineages of the Cryptomycota and Microsporidia. Yeast-like fungi tended to have the highest number of proteins. Some remarkable patterns of high sequence similarity throughout the tree were seen for Cdc42, Rdi1, Sec4, and Rho3. A possible explanation for this observation is that all of these proteins, with the exception of Rdil, are essential proteins-albeit in Saccharomyces cerevisiae - and that all four proteins are short proteins (i.e. $<250$ amino acids) and consist of a single domain. It has been hypothesized that basically all residue sites are functionally important and that selection is strong to prevent deleterious mutations. Another interesting factor is that we found a group of 16 proteins with a high prevalence in all ascomycetes and basidiomycetes. This group includes $\mathrm{Cdc} 42$ and its direct regulators and effectors, and also proteins such as Spa2, Rho3 and Sec4/15. Interestingly, over 95\% of the species examined contained at least $75 \%$ of this core. We therefore hypothesize that polarity establishment by regulation of Cdc42 is conserved over the tree of life, but the set of proteins that performs this function varies between species.

\section{Concluding remarks: polarization protein network and organismal diversity}

In this synopsis we focus on the evolutionary dynamics and the mechanisms underlying the polarization network across fungi. Much work has been performed in order to work towards a complete understanding of this highly complex protein network. But how far have we come? And what needs to be done to be able to mechanistically understand the observed conservation and variability of the network across species? 
Ultimately, we want to be able to link the profound amount of knowledge currently available to the biology of individual species. This means that we want to be able to explain a species' morphology, physiology and ecology using the results of bio-informatics, proteomics, functional, experimental evolution and theoretical modeling studies.

To date, most of the work has been done in the budding yeast Saccharomyces cerevisiae in a wide array of fields. This species has become the ultimate model system to study the molecular key players and underlying mechanism of cell polarization. Influential studies that started describing normal budding behavior, polarity of cellular content and the effect of mutations on polarization and abnormal budding patterns started roughly five decades ago (Marchant and Smith 1968; Sloat and Pringle 1978; Borisy 1978). Subsequent decades were witness to progression in determining the members of the protein network (discussed in the section The polarization machinery of fungal model systems), the functional importance of individual proteins through, for example, knock-out studies (Arkowitz and Lowe 1997; Bi and Park 2012) and, more recently, the essentiality and/or evolvability of protein network members (Laan et al. 2015; Liu et al. 2015) and theoretical modeling of the regulatory mechanisms of the pathway (see Box 1 in the Appendix). Despite these overwhelming number of studies, even within $S$. cerevisiae questions remain unanswered. For example, not much is known on how the $S$. cerevisiae protein network adapts to changes to coremembers of the network. We do know the phenotypic effects of knock-outs of all $S$. cerevisiae genes through the generation of tools such as the Yeast Deletion Project (Giaever et al. 2002). However, an increasing number of studies indicate that the essentiality of a gene is context dependent; a gene can be essential under one growth condition but not in another. Also, importantly, due to epistasis the essentiality of a gene can be dependent on the genetic background. Although tools such as the Biological General Repository for Interaction Datasets (thebiogrid.org) contain a vast amount of data on interactions between loci, and some insights on epistasis are available (Jasnos and Korona 2007), the complex interactions of loci under experimental or natural settings are still hard to predict. In order to further understand the evolvability of the network, experimental studies are needed to provide new insights. The study by Laan et al. (2015) also identified a locus with so far unidentified functions in the polarization network: Nrp1. This locus was found to be inactivated, and the authors hypothesized a role in the process of initiating polarization (Laan et al. 2015). This finding indicates that even in $S$. cerevisiae not all (indirect) players of $\mathrm{Cdc} 42$ regulation have been discovered. Ultimately, this means that even after five decades of work the polarization protein network and especially its epigenetic interactions are still not entirely uncovered in S. cerevisiae.

Other complexities arise with comparative genomics studies. Care should be taken when homologs are identified based on sequence similarity. Even when a species has a true ortholog to a Saccharomyces cerevisiae locus with demonstrated functions in the polarization in budding yeast, orthologous functions cannot be assumed. With the increased availability of genomic and proteomic datasets [e.g. deletion libraries (Giaever et al. 2002); the Saccharomyces Genome Database (SGD; Cherry et al. 2011)] it is becoming more feasible to perform large-scale comparative screens. Therefore, the development of sophisticated tools to reliably identify orthologs is essential. Furthermore, any findings of orthologs need to be functionally confirmed, as proteins with shared ancestry can have different functions or can be used differently in different species; for example, Cdc42 is not the master regulator of polarity in Ustilago maydis (Mahlert et al. 2005). Different fungal species are also characterized by entirely different groups of essential proteins (Seiler and Plamann 2003). Further, functional studies are indispensable because fully sequenced fungal genomes are frequently characterized by a relatively high level of loci without homologs in available fungal databases (Galagan et al. 2003). Such loci are not taken into account in most comparative studies. Functional screening of individual (non-model) species would therefore provide valuable information about such species- or lineagespecific proteins and adaptation.

The observation that different species make use of proteins in different ways may be seen as a sign of adaptive (protein) evolution. Each species has to constantly adapt to its currentand often changing - environment. Included in the fungal kingdom are species adapted to a highly diverse array of environments, from forest soil decayers to plant- or vertebratespecialized parasites. The wide array of different specialists resembles the wide array of niches filled by fungi. With niche specialization comes diversifying morphologies. There are an estimated of up to 5.1 million fungal species (O'Brien et al. 2005), including different types of unicellular and multicellular organisms (Fig. 2), and species that switch back and forth between two or more lifestyles. Morphologies are highly distinct within each different lifestyle or lifephase; for example, the fruiting bodies of basidiomycetes (i.e., mushrooms) vary greatly in shape, size and color, and there is a great variety in shape and mode of division in unicellular fungi ( budding yeast, fission yeast, Microsporidia). So how can we link or explain the level of phenotypic diversity with patterns of variation and conservation, sequence similarity, identified orthologs and functional discrepancies in the polarization network? What part does the mode of polarization play in the adaptation of a species to its niche? If we want to be able to understand why this protein network comprises different members in different species and acts differently in different organisms, then we also need to understand why their morphologies are different.

Various aspects of the fungal diverse life strategies make it hard to link the polarization repertoire with specific phenotypes. Many different species are bi- or trimorphic, meaning 
that they can switch back and forth between unicellular to multicellular states depending on various environmental stimuli and/or stressors. The cell shapes of these different states are highly dissimilar and seem to be related to the specific environment the cell is in. For example, in nutrient-rich media, Saccharomyces cerevisiae grows by budding, while in nutrient-poor conditions they grow pseudohyphae. Furthermore, one big difference between the different life phases or morphologies is that cell growth by budding and pseudohyphae is cell cycle dependent, while hyphal growth is not. This means that there is continuous polarized growth in the hyphae, without cell separation, while polarized growth in yeast and pseudohyphae only takes place in the G1 phase of the cell cycle, followed by cell separation. This decoupling of polarized growth and cell cycle suggests a relaxed need of cyclin-dependent kinases, as Cdc28 is essential in S. cerevisiae (Liu et al. 2015). In addition, filamentous fungi do not need the S. cerevisiae chitin ring, which forms the site of cell separation, nor the associated proteins. A further distinction can be made in growth speed of filamentous fungi and the mechanism they use to polarize the cytoskeleton. Fastgrowing fungi, such as Ustilago maydis and Neurospora crassa depend on the actin cytoskeleton plus microtubule dynamics, while the slower growing filamentous fungi Ashbya gossypii depends more on the actin cytoskeleton, as discussed by Arkowitz and Bassilana (2011). These different methods imply differences in sets of proteins regulating the specific mode of cytoskeleton organization.

Table 1 reveals some signs that there are a couple of yeastspecific polarization proteins, such as Gic $1 / 2$ and Bem 4 , and also of some putative filamentous-specific polarization proteins not found in unicellular fungi, such as Ro1/3/10 in Neurospora crassa. At the same time, not all unicellular or filamentous fungi possess these proteins, indicating that they are not crucial to that particular morphology. In fact, virtually all species seem to make use of a general pathway in which (1) a pre-existing or external signal leads to polarity establishment by GTPase(s) controlled by several regulators, effectors and scaffolding proteins, (2) the cytoskeleton is polarized, induced by the members of a polarisome and (3) exocytosis and endocytosis are needed at the site of growth (Fig. 1). The exact proteins that perform roles within these processes and feedback loops can differ and can be seen as a species' adaptation. However, a number of proteins are present across species, and these proteins seem to be the (flexible) core of polarization (Diepeveen et al. 2017). This core does not appears to be specific to life stage or morphology as the proteins forming this core are found across species with different morphologies.

In conclusion, the evolutionary dynamics of the fungal polarization protein network include both a shared mechanism, represented by a core of shared proteins, and variation in the presence and/or importance of processes and network members that can sometimes be linked to function/phenotype. This conserved core of proteins offers opportunities to study the evolutionary dynamics of the polarization network in more detail, such as by testing orthology functionally. However, since the bulk of work has been performed in a handful of developed fungal model systems, which often are separated by high levels of divergence and have quite different lifestyles, validation of suggested patterns between the mechanism of polarization and morphology is needed. We urge for a deeper investigation of functional characterization in a wider variety of species and for further development of strong/reliable computational tools to support future large-scale comparative studies. The last five decades of research on the fungal polarization network have resulted in signals that the variability of the protein network might reflect the great diversity in lifestyles/morphologies observed in fungi. However, broader comparative approaches are needed to test the generality of these observations in order to be able to link the polarization networks to the species' biology.

Acknowledgments We wish to thank WKG Daalman for providing valuable feedback and discussion on this manuscript. We apologize to our colleagues' work that did not get cited here due to space restrictions. We would also like to thank two anonymous reviewers for their constructive comments. This work was supported by the Netherlands Organization for Scientific Research (NWO/OCW), as part of the Frontiers of Nanoscience program to Liedewij Laan and a TU Delft Health Scholarship to Leila Iñigo de la Cruz.

\section{Compliance with ethical standards}

Conflicts of interest Eveline T. Diepeveen, Leila Iñigo de la Cruz and Liedewij Laan declare that they have no conflicts of interest.

Ethical approval This article does not contain any studies with human participants or animals performed by any of the authors.

\section{Appendix 1: Box 1-theoretical molecular models of yeast cell polarity}

Much progress has been made on the development of theoretical molecular models of yeast cell polarity. Here we give a brief introductory and non-exhaustive overview of the most commonly used models. For a broad and detailed discussion of theoretical modeling of shape and growth we refer the reader to Pelcé (2004). The aim of theoretical models is to describe spontaneous symmetry breaking during yeast polarization based on positive feedback loops for the activation and localization of $\mathrm{Cdc} 42$. These feedback loops are required to destabilize a spatially uniform state of the cell (Goryachev and Leda 2017). From the field of physics, we know that spontaneous symmetry breaking is achieved when the symmetric state is no longer stable. In the case of cell polarity, the behavior is very close to equilibrium phase transitions, where there is a critical value - for example, the cytoplasmic concentration of $\mathrm{Cdc} 42$ activator (Gulli et al. 2000) and/or Cdc42 inhibitor activity 
(Knaus et al. 2007), beyond which the spatially homogeneous state is unstable (Lo et al. 2014). Cdc42-GTP clusters have become the de facto criterion for judging whether a given set of experimental conditions allow or prevent cellular polarization. Historically, $\mathrm{Cdc} 42$ polarization in budding yeast has been described by two categorized pathways, namely, the actin pathway and the reaction-diffusion pathway. The actin pathway describes the polarization of $\mathrm{Cdc} 42$ mediated by protein transport on actin cables. However, as the exact cargo and function of the pathway is still under debate (Layton et al. 2011; Freisinger et al. 2013; Watson et al. 2014), constructing a realistic model for symmetry breaking via this pathway has been complicated. In the reaction-diffusion pathway, the spontaneous symmetry breaking conditions are fulfilled (Goryachev and Leda 2017) and can be linked to Cdc42 polarization in yeast cells (Irazoqui et al. 2003). Landmarks and sensory inputs can be added as spatially dependent initial conditions (for a review, see Chang and Peter 2003).

The necessary condition of a model that attempts to describe the spontaneous Cdc42 polarization in yeast is the ability to maintain a kind of "memory" from the initial condition by sustaining polarization long after the initial perturbation. To accomplish that condition, it is necessary that the rate of activation grows faster with increasing Cdc42-GTP than does the rate of inactivation (Goryachev and Leda 2017), assuming that the total cellular amount of $\mathrm{Cdc} 42$ is conserved during the transition. Theoretically, the only way to ensure this condition is by having multiple cooperating feedback loops, for example by having the local rates of activation/inactivation depend on the activity of Cdc42 itself (Lo et al. 2014). The mathematics behind the modeling approach always involves solving a coupled system of reaction diffusion equations, and a stability analysis of the steady state solution:

$$
\begin{aligned}
& \frac{\partial P_{1}}{\partial t}(x, t)=f_{1}\left(P_{1}(x, t), P_{2}(x, t), \ldots, P_{n}(x, t), x, t ; k_{1}, k_{2}, \ldots, k_{m}\right)+D_{1} P_{1 x x} \\
& \vdots \vdots \\
& \frac{\partial P_{n}}{\partial t}(x, t)=f_{n}\left(P_{n}(x, t), \ldots, P_{1}(x, t), P_{2}(x, t), x, t ; k_{1}, k_{2}, \ldots, k_{m}\right)+D_{n} P_{n x x}
\end{aligned}
$$

where, $P(x, t)$ is the time-evolution of the concentrations of the different proteins, which is given by reaction kinetics, $D_{P}$ is a diagonal matrix encoding the diffusion constants for the proteins $P(x, t), x$ is a spatial coordinate, $t$ is time and $k_{1}, k_{2}, \ldots$, $k_{n}$ are the reaction-rate constants. $f$ is a function that describes the local reactions. The term $P_{n x x}$ refers to the second spatial derivative of the $n$-th protein concentration. Symmetry breaking is modeled in a spatially extended system accounting for the membrane as well as the cytosol. Different spatial dimensions are used to model the reaction diffusions equations according to how the proteins diffuse; for example, along a curve (one-dimension) (Smith et al. 2013), membrane surface (two dimensions) (Thalmeier et al. 2016) or cytoplasm (three dimensions) (Klünder et al. 2013).
Experimental cell biology studies (Kozubowski et al. 2008), the evolution of the polarity network as well as theoretical studies suggest that several feedback loops are simultaneously at play (Goryachev and Leda 2017) and that only a few mutations are necessary to switch between them (Laan et al. 2015).

Open Access This article is distributed under the terms of the Creative Commons Attribution 4.0 International License (http:// creativecommons.org/licenses/by/4.0/), which permits unrestricted use, distribution, and reproduction in any medium, provided you give appropriate credit to the original author(s) and the source, provide a link to the Creative Commons license, and indicate if changes were made.

\section{References}

Arkowitz RA, Bassilana M (2011) Polarized growth in fungi: symmetry breaking and hyphal formation. Semin Cell Dev Biol 22:806-815. doi:10.1016/j.semcdb.2011.08.010

Arkowitz RA, Lowe N (1997) A small conserved domain in the yeast Spa2p is necessary and sufficient for its polarized localization. J Cell Biol 138:17-36

Banuett F, Quintanilla RH Jr, Reynaga-Peña CG (2008) The machinery for cell polarity, cell morphogenesis, and the cytoskeleton in the Basidiomycete fungus Ustilago Maydis - a survey of the genome sequence. Fungal Genet Biol 45:S3-S14. doi:10.1016/j.fgb.2008. 05.012

Bauer Y, Knechtle P, Wendland J et al (2004) A Ras-like GTPase is involved in hyphal growth guidance in the filamentous fungus Ashbya gossypii. Mol Biol Cell 15:4622-4632. doi:10.1091/mbc. E04-02-0104

Bender A, Pringle JR (1989) Multicopy suppression of the cdc24 budding defect in yeast by CDC42 and three newly identified genes including the ras-related gene RSR1. Proc Natl Acad Sci USA 86:99769980

Bender L, Lo HS, Lee H et al (1996) Associations among PH and SH3 domain-containing proteins and rho-type GTPases in yeast. J Cell Biol 133:879-894

Bi E, Park HO (2012) Cell polarization and cytokinesis in budding yeast. Genetics 191:347-387. doi:10.1534/genetics.111.132886

Borisy GG (1978) Polarity of microtubules of the mitotic spindle. J Mol Biol 124:565-570

Boynton PJ, Greig D (2016) Species richness influences wine ecosystem function through a dominant species. Fungal Ecol 22:61-72. doi:10. 1016/j.funeco.2016.04.008

Brown JL, Jaquenoud M, Gulli MP et al (1997) Novel Cdc42-binding proteins Gic1 and Gic2 control cell polarity in yeast. Genes Dev 11: 2972-2982

Browning H, Hayles J, Mata J et al (2000) Tea2p is a kinesin-like protein required to generate polarized growth in fission yeast. J Cell Biol $151: 15-27$

Brunner D, Nurse P (2000) CLIP170-like tip1p spatially organizes microtubular dynamics in fission yeast. Cell 102:695-704

Chang EC, Barr M, Wang Y et al (1994) Cooperative interaction of S. pombe proteins required for mating and morphogenesis. Cell 79: 131-141

Chang F, Peter M (2003) Yeasts make their mark. Nat Cell Biol 5:294299. doi:10.1038/ncb0403-294

Chant J (1999) Cell polarity in yeast. Annu Rev Cell Dev Biol 15:365391. doi:10.1146/annurev.cellbio.15.1.365 
Chant J, Herskowitz I (1991) Genetic control of bud site selection in yeast by a set of gene products that constitute a morphogenetic pathway. Cell 65:1203-1212

Chant J, Corrado K, Pringle JR, Herskowitz I (1991) Yeast BUD5, encoding a putative GDP-GTP exchange factor, is necessary for BUD site selection and interacts with bud formation gene BEM1. Cell 65:1213-1224

Chen GC, Kim YJ, Chan C (1997) The Cdc42 GTPase-associated proteins Gic1 and Gic2 are required for polarized cell growth in Saccharomyces cerevisiae. Genes Dev 11:2958-2971

Chen GC, Zheng L, Chan CS (1996) The LIM domain-containing Dbm1 GTPase-activating protein is required for normal cellular morphogenesis in Saccharomyces cerevisiae. Mol Cell Biol 16:1376-1390

Chen T, Hiroko T, Chaudhuri A et al (2000) Multigenerational cortical inheritance of the Rax 2 protein in orienting polarity and division in yeast. Science 290:1975-1978

Chenevert J, Corrado K, Bender A et al (1992) A yeast gene (Bem1) necessary for cell polarization whose product contains $2 \mathrm{Sh} 3$ domains. Nature 356:77-79. doi:10.1038/356077a0

Cherry JM, Hong EL, Amundsen C et al (2011) Saccharomyces genome database: the genomics resource of budding yeast. Nucleic Acids Res 40:D700-D705. doi:10.1093/nar/gkr1029

Coll PM, Trillo Y, Ametzazurra A, Perez P (2003) Geflp, a new guanine nucleotide exchange factor for $\mathrm{Cdc} 42 \mathrm{p}$, regulates polarity in Schizosaccharomyces pombe. Mol Biol Cell 14:313-323. doi:10. 1091/mbc.E02

Cotteret S, Chernoff J (2002) The evolutionary history of effectors downstream of Cdc42 and Rac. Genome Biol 3(2):REVIEWS0002

Diepeveen ET, Pourquié V, Gehrmann T et al (2017) Different levels of protein conservation in the fungal polarization network. BioRxiv 154641. doi: 10.1101/154641

Drees BL, Sundin B, Brazeau E et al (2001) A protein interaction map for cell polarity development. J Cell Biol 154:549-571

Drubin DG (2000) Cell polarity. Oxford University Press, Oxford

Evangelista M, Blundell K, Longtine MS et al (1997) Bnilp, a yeast formin linking Cdc42p and the actin cytoskeleton during polarized morphogenesis. Science 276:118-122

Evlampiev K, Isambert H (2008) Conservation and topology of protein interaction networks under duplication-divergence evolution. Proc Natl Acad Sci USA 105:9863-9868. doi:10.1073/pnas.0804119105

Feierbach B, Chang F (2001) Cytokinesis and the contractile ring in fission yeast. Curr Opin Microbiol 4:713-719

Freisinger T, Klünder B, Johnson J et al (2013) Establishment of a robust single axis of cell polarity by coupling multiple positive feedback loops. Nat Commun 4:1807-1811. doi:10.1038/ncomms2795

Fujita A, Lord M, Hiroko T et al (2004) Rax1, a protein required for the establishment of the bipolar budding pattern in yeast. Gene 327: 161-169. doi:10.1016/j.gene.2003.11.021

Fujita A, Oka C, Arikawa Y et al (1994) A yeast gene necessary for budsite selection encodes a protein similar to insulin-degrading enzymes. Nature 372:567-570. doi:10.1038/372567a0

Galagan JE, Calvo SE, Borkovich KA et al (2003) The genome sequence of the filamentous fungus Neurospora crassa. Nature 422:859-868. doi:10.1038/nature 01554

Giaever G, Chu AM, Ni L et al (2002) Functional profiling of the Saccharomyces cerevisiae genome. Nature 418:387-391. doi:10. 1038/nature00935

Goldstein B, Macara IG (2007) The PAR proteins: fundamental players in animal cell polarization. Dev Cell 13:609-622. doi:10.1016/j. devcel.2007.10.007

Goryachev AB, Leda M (2017) Many roads to symmetry breaking: molecular mechanisms and theoretical models of yeast cell polarity. Mol Biol Cell 28:370-380. doi:10.1091/mbc.E16-10-0739

Gresham D, Desai MM, Tucker CM et al (2008) The repertoire and dynamics of evolutionary adaptations to controlled nutrient-limited environments in yeast. PLoS Genet 4:e1000303. doi:10.1371/ journal.pgen.1000303.s014

Gulli MP, Jaquenoud M, Shimada Y et al (2000) Phosphorylation of the Cdc42 exchange factor $\mathrm{Cdc} 24$ by the PAK-like kinase Cla4 may regulate polarized growth in yeast. Mol Cell 6:1155-1167

Halme A, Michelitch M, Mitchell EL, Chant J (1996) Bud10p directs axial cell polarization in budding yeast and resembles a transmembrane receptor. Curr Biol 6:570-579

Harris SD (2006) Cell polarity in filamentous fungi: shaping the mold. Int Rev Cytol 251:41-77. doi:10.1016/S0074-7696(06)51002-2

Herskowitz I (1995) MAP kinase pathways in yeast: for mating and more. Cell 80:187-197

Inouye C, Dhillon N, Thorner J (1997) Ste5 RING-H2 domain: role in Ste4-promoted oligomerization for yeast pheromone signaling. Science 278:103-106

Irazoqui JE, Gladfelter AS, Lew DJ (2003) Scaffold-mediated symmetry breaking by Cdc42p. Nat Cell Biol 5:1062-1070. doi:10.1083/jcb. 142.5 .1301

Jackson SL, Heath IB (1993) Roles of calcium ions in hyphal tip growth. Microbiol Rev 57:367-382

Jasnos L, Korona R (2007) Epistatic buffering of fitness loss in yeast double deletion strains. Nat Genet 39:550-554. doi:10.1038/ng1986

Johnson DI (1999) Cdc42: an essential rho-type GTPase controlling eukaryotic cell polarity. Microbiol Mol Biol Rev 63:54-105

Kang PJ, Lee ME, Park H-O (2014) Bud3 activates Cdc42 to establish a proper growth site in budding yeast. J Cell Biol 206:19-28. doi:10. 1083/jcb.201402040.dv

Kaufmann A (2008) Polarized growth and septation in the filamentous ascomycete Ashbya gossypii analyzed by live cell imaging. $\mathrm{PhD}$ thesis. University of Basel, Basel

Kemphues KJ, Priess JR, Morton DG, Cheng C (1988) Identification of genes required for cytoplasmic localization in early C-Elegans embryos. Cell 52:311-320

Kim YJ, Francisco L, Chen GC et al (1994) Control of cellular morphogenesis by the Ip12/Bem2 GTPase-activating protein: possible role of protein phosphorylation. J Cell Biol 127:1381-1394

Klünder B, Freisinger T, Wedlich-Söldner R, Frey E (2013) GDImediated cell polarization in yeast provides precise spatial and temporal control of Cdc42 signaling. PLoS Comput Biol 9:e1003396. doi:10.1371/journal.pcbi.1003396.s008

Knaus M, Pelli-Gulli M-P, van Drogen F et al (2007) Phosphorylation of Bem $2 p$ and Bem $3 p$ may contribute to local activation of Cdc $42 p$ at bud emergence. EMBO J 26:4501-4513. doi:10.1038/sj.emboj. 7601873

Köhli M, Gelati V, Boudier K et al (2008) Growth-speed-correlated localization of exocyst and polarisome components in growth zones of Ashbya gossypii hyphal tips. J Cell Sci 121:3878-3889. doi:10. 1242 jes.033852

Kozubowski L, Saito K, Johnson JM et al (2008) Symmetry-breaking polarization driven by a Cdc42p GEF-PAK complex. Curr Biol 18:1719-1726. doi:10.1016/j.cub.2008.09.060

Laan L, Koschwanez JH, Murray AW (2015) Evolutionary adaptation after crippling cell polarization follows reproducible trajectories. elife 4:e09638. doi:10.7554/eLife.09638

Layton AT, Savage NS, Howell AS et al (2011) Modeling vesicle traffic reveals unexpected consequences for Cdc42p-mediated polarity establishment. Curr Biol 21:184-194. doi:10.1016/j.cub.2011.01.012

Lee C, Occhipinti P, Gladfelter AS (2015) PolyQ-dependent RNA-protein assemblies control symmetry breaking. J Cell Biol 208:533544. doi:10.1016/S0378-1119(99)00509-0

Leeuw T, Wu CL, Schrag JD et al (1998) Interaction of a G-protein betasubunit with a conserved sequence in Ste20/PAK family protein kinases. Nature 391:191-195. doi:10.1038/34448

Leu J-Y, Murray AW (2006) Experimental evolution of mating discrimination in budding yeast. Curr Biol 16:280-286. doi:10.1016/j.cub. 2005.12.028 
Lew DJ, Reed SI (1993) Morphogenesis in the yeast cell cycle: regulation by Cdc28 and cyclins. J Cell Biol 120:1305-1320

Li R, Bowerman B (2010) Symmetry breaking in biology. Cold Spring Harb Perspect Biol 2:a003475-a003475. doi:10.1101/cshperspect. a003475

Li SJ, Du LC, Yuen G, Harris SD (2006) Distinct ceramide synthases regulate polarized growth in the filamentous fungus Aspergillus nidulans. Mol Biol Cell 17:1218-1227. doi:10.1091/mbc.E05-060533

Liao Y, He F, Gong T et al (2013) Msb1 interacts with Cdc42, Boil, and Boi2 and may coordinate Cdc42 and Rho1 functions during early stage of bud development in budding yeast. PLoS One 8:e66321. doi:10.1371/journal.pone.0066321.s003

Lin M, Unden H, Jacquier N et al (2009) The Cdc42 effectors Ste20, Cla4, and Skm1 down-regulate the expression of genes involved in sterol uptake by a mitogen-activated protein kinase-independent pathway. Mol Biol Cell 20:4826-4837. doi:10.1091/mbc.E09-010034

Liu G, Yong MYJ, Yurieva M et al (2015) Gene essentiality is a quantitative property linked to cellular evolvability. Cell 163:1388-1399. doi:10.1016/j.cell.2015.10.069

Lo W-C, Park H-O, Chou C-S (2014) Mathematical analysis of spontaneous emergence of cell polarity. Bull Math Biol 76:1835-1865. doi:10.1007/s11538-014-9982-2

Machesky LM, Atkinson SJ, Ampe C et al (1994) Purification of a cortical complex containing two unconventional actins from Acanthamoeba by affinity-chromatography on profilin-agarose. J Cell Biol 127:107-115

Mahlert M, Leveleki L, Hlubek A et al (2005) Rac1 and Cdc42 regulate hyphal growth and cytokinesis in the dimorphic fungus Ustilago maydis. Mol Microbiol 59:567-578. doi:10.1111/j.1365-2958. 2005.04952.x

Marchant R, Smith DG (1968) Bud formation in Saccharomyces cerevisiae and a comparison with the mechanism of cell division in other yeasts. J Gen Microbiol 53:163-169. doi:10.1099/ 00221287-53-2-163

Martin SG (2015) Spontaneous cell polarization: feedback control of Cdc42 GTPase breaks cellular symmetry. BioEssays 37:11931201. doi:10.1002/bies.201500077

Martin SG, Arkowitz RA (2014) Cell polarization in budding and fission yeasts. FEMS Microbiol Rev 38:228-253. doi:10.1111/1574-6976. 12055

Matsui Y, Matsui R, Akada R, Toh-e A (1996) Yeast src homology region 3 domain-binding proteins involved in bud formation. J Cell Biol $113: 865-878$

Nakano K, Imai J, Arai R et al (2002) The small GTPase Rho3 and the diaphanous/formin For3 function in polarized cell growth in fission yeast. J Cell Sci 115:4629-4639. doi:10.1242/jcs.00150

Nakano K, Toya M, Yoneda A et al (2011) Pob1 ensures cylindrical cell shape by coupling two distinct rho signaling events during cecretory vesicle targeting. Traffic 12:726-739. doi:10.1111/j.1600-0854. 2011.01190.x

Nelson WJ (2003) Adaptation of core mechanisms to generate cell polarity. Nature 422:766-774. doi:10.1038/nature01602

Nishimura T, Yamaguchi T, Tokunaga A et al (2006) Role of numb in dendritic spine development with a Cdc42 GEF intersectin and EphB2. Mol Biol Cell 17:1273-1285. doi:10.1091/mbc.E05

O'Brien HE, Parrent JL, Jackson JA et al (2005) Fungal community analysis by large-scale sequencing of environmental samples. Appl Environ Microbiol 71:5544-5550. doi:10.1128/AEM.71.9.55445550.2005

Park HO, Bi E (2007) Central roles of small GTPases in the development of cell polarity in yeast and beyond. Microbiol Mol Biol Rev 71:48 96. doi:10.1128/MMBR.00028-06
Park HO, Chant J, Herskowitz I (1993) Bud2 encodes a Gtpase-activating protein for Bud1/Rsr1 necessary for proper bud-site selection in yeast. Nature 365:269-274. doi:10.1038/365269a0

Park HO, Bi EF, Pringle JR, Herskowitz I (1997) Two active states of the Ras-related Bud1/Rsr1 protein bind to different effectors to determine yeast cell polarity. Proc Natl Acad Sci USA 94:4463-4468

Pál C, Papp B, Lercher MJ (2006) An integrated view of protein evolution. Nat Rev Genet 7:337-348. doi:10.1038/nrg1838

Pelcé P (2004) New visions on form and growth: fingered growth, dendrites and flames. Oxford University Press, Oxford

Pelham RJ, Chang F (2002) Actin dynamics in the contractile ring during cytokinesis in fission yeast. Nature 419:82-86. doi:10.1038/ nature00999

Peter M, Herskowitz I (1994) Direct inhibition of the yeast cyclindependent kinase Cdc28-Cln by Far1. Science 265:1228-1231. doi:10.1126/science.8066461

Philippsen P, Kaufmann A, Schmitz H-P (2005) Homologues of yeast polarity genes control the development of multinucleated hyphae in Ashbya gossypii. Curr Opin Microbiol 8:370-377. doi:10.1016/j. mib.2005.06.021

Pringle JR, Bi E, Harkins HA et al (1995) Establishment of cell polarity in yeast. Cold Spring Harb Symp Quant Biol 60:729-744

Pruyne D, Bretscher A (2000) Polarization of cell growth in yeast. I. Establishment and maintenance of polarity states. J Cell Sci 113: 365-375

Pruyne D, Legesse-Miller A, Gao L et al (2004) Mechanisms of polarized growth and organelle segregation in yeast. Annu Rev Cell Dev Biol 20:559-591. doi:10.1146/annurev.cellbio.20.010403.103108

Pryciak PM, Huntress FA (1998) Membrane recruitment of the kinase cascade scaffold protein Ste 5 by the $\mathrm{G}$ beta gamma complex underlies activation of the yeast pheromone response pathway. Genes Dev 12:2684-2697

Rincon SA, Ye Y, Villar-Tajadura MA et al (2009) Pob1 participates in the $\mathrm{Cdc} 42$ regulation of fission yeast Actin cytoskeleton. Mol Biol Cell 20:4390-4399. doi:10.1091/mbc.E09-03-0207

Riquelme M, Yarden O, Bartnicki-Garcia S et al (2011) Architecture and development of the. Fungal Biol 115:446-474. doi:10.1016/j. funbio.2011.02.008

Robinson N, Guo L, Imai J et al (1999) Rho3 of Saccharomyces cerevisiae, which regulates the actin cytoskeleton and exocytosis, is a GTPase which interacts with Myo2 and Exo70. Mol Cell Biol 19:3580-3587

Roemer T, Madden K, Chang JT, Snyder M (1996) Selection of axial growth sites in yeast requires Axl2p, a novel plasma membrane glycoprotein. Genes Dev 10:777-793

Segrè AV, Murray AW, Leu JY (2006) High-resolution mutation mapping reveals parallel experimental evolution in yeast. PLoS Biol 4:e256. doi:10.1371/journal.pbio.0040256

Seiler S, Plamann M (2003) The genetic basis of cellular morphogenesis in the filamentous fungus Neurospora crassa. Mol Biol Cell 1-11. doi: 10.1091/mbc.E02

Shimada Y, Gulli MP, Peter M (2000) Nuclear sequestration of the exchange factor Cdc24 by Far1 regulates cell polarity during yeast mating. Nat Cell Biol 2:117-124. doi:10.1038/35000073

Sloat BF, Pringle JR (1978) A mutant of yeast defective in cellular morphogenesis. Science 200:1171-1173

Smith GR, Givan SA, Cullen P, Sprague GF (2002) GTPase-activating proteins for Cdc42. Eukaryot Cell 1:469-480. doi:10.1128/EC.1.3. 469-480.2002

Smith SE, Rubinstein B, Mendes Pinto I et al (2013) Independence of symmetry breaking on Bem1-mediated autocatalytic activation of Cdc42. J Cell Biol 202:1091-1106. doi:10.1074/jbc.270.2.626

Stevenson BJ, Ferguson B, De Virgilio C et al (1995) Mutation of RGA1, which encodes a putative GTPase-activating protein for the polarityestablishment protein $\mathrm{Cdc} 42 \mathrm{p}$, activates the pheromone-response 
pathway in the yeast Saccharomyces cerevisiae. Genes Dev 9:29492963

Thalmeier D, Halatek J, Frey E (2016) Geometry-induced protein pattern formation. Proc Natl Acad Sci USA 113:548-553. doi:10.1083/jcb. 201403136

Watson LJ, Rossi G, Brennwald P (2014) Quantitative analysis of membrane trafficking in regulation of Cdc42 polarity. Traffic 15:1330 1343. doi:10.1111/tra. 12211

Wendland J (2001) Comparison of morphogenetic networks of filamentous fungi and yeast. Fungal Genet Biol 34:63-82. doi:10.1006/fgbi. 2001.1290

Wendland J, Philippsen P (2001) Cell polarity and hyphal morphogenesis are controlled by multiple rho-protein modules in the filamentous ascomycete Ashbya gossypii. Genetics 157:601-610

Wendland J, Philippsen P (2000) Determination of cell polarity in germinated spores and hyphal tips of the filamentous ascomycete Ashbya gossypii requires a rhoGAP homolog. J Cell Sci 113:1611-1621
Whiteway M, Wu C, Leeuw T et al (1995) Pheromone response in yeastassociation of Bem $1 \mathrm{p}$ with proteins of the map Kinase Cascade and Actin. Science 270:1210-1213

Zahner JE, Harkins HA, Pringle JR (1996) Genetic analysis of the bipolar pattern of bud site selection in the yeast Saccharomyces cerevisiae. Mol Cell Biol 16:1857-1870

Zhang J, Yang J-R (2015) Determinants of the rate of protein sequence evolution. Nat Rev Genet 16:409-420. doi:10.1038/nrg3950

Zheng Y, Bender A, Cerione R (1995) Interactions among proteins involved in bud-site selection and bud-site assembly in Saccharomyces cerevisiae. J Biol Chem 270:626-630

Zheng Y, Cerione R, Bender A (1994) Control of the yeast bud-site assembly Gtpase Cdc42 - catalysis of guanine-nucleotide exchange by Cdc 24 and stimulation of Gtpase activity by Bem3. J Biol Chem 269:2369-2372 